\title{
Restricted magnetic resonance diffusion of lung consolidation is not specific for respiratory exacerbation
}

\author{
To the Editor:
}

We read with interest the recent publication by CIET et al. [1] related to the diagnosis of respiratory tract exacerbation (RTE) in cystic fibrosis (CF) using diffusion-weighted magnetic resonance imaging (DWI). RTE is a difficult diagnosis and their results highlight the usefulness of magnetic resonance imaging (MRI) in radiation-free management of CF. In this study, the visual analysis of a DWI score had good diagnostic accuracy to discriminate between controls and RTE's and the authors must be congratulated for this result. However, in their study, the total acquisition time in the supine position to complete both T2 and DWI sequences was $30 \mathrm{~min}$, which is very long in the clinical context of RTE if we are to obtain artefact-free images. In addition, only the largest restricted consolidations in the RTE group were selected for analysis, meaning that bronchi potentially thickened and mucus-filled during RTE were excluded. Last but not least, in order to assess the value of DWI as compared to a standard T2 sequence for diagnosing RTE, it is necessary to demonstrate whether or not the DWI "hotspots" could correspond to an artefact called the T2-shine-through effect [2]. Indeed, T2 has already been demonstrated as a biomarker for acute lung inflammation [3]. To solve this, the authors performed quantitative measurements of the apparent diffusion coefficient (ADC) in both controls and subjects undergoing RTE. In controls, ADC measurements were performed randomly inside the lung parenchyma when no hotspots were visible. Surprisingly, the receiver operating characteristic (ROC) curves of the ADC indicated $100 \%$ specificity for the diagnosis of RTE towards the lowest ADC values. This crucial point is unfortunately not discussed though it is a feature that corresponds neither to the literature [4-6] nor to the physical principle of this measurement. Indeed, the general mathematical formula to calculate an ADC value is: $A D C=-1 / b_{1} \cdot \operatorname{Ln}\left(S_{1} / S_{0}\right)$ where $S_{1}$ and $S_{0}$ correspond to the DWI signals at two b-values, where $b$ is the gradient factor. Therefore, there are two mathematical possibilities under normal conditions: 1) Owing to the very short decay time of the lung signal due to susceptibility artefacts, there is a need for ultra-short echo times (of a microsecond order of magnitude) in order to obtain any signal from the lungs [7, 8]. The DWI echo time was 54 ms and thus the lung signal was a null value at all $b$-values. In agreement with this, the figures in the article display no vessel and no signal inside the lung (and, as a consequence, $S_{1}=S_{0}=0$ ). 2) Due to additional noise, $S_{1}$ and $S_{0}$ may not be exactly equal to zero (as can also be seen in the figures of the article). Therefore $S_{1}=\left(s_{1}+n_{1}\right)$ and $S_{0}=\left(s_{0}+n_{0}\right)$, where $n$ represents the level of noise. Since noise is expected to be constant $\mathrm{n}_{0} \approx \mathrm{n}_{1}$ and, therefore, the mathematical formula for $\mathrm{ADC}$ becomes: $\mathrm{ADC}=-1 / \mathrm{b} \cdot \operatorname{Ln}(1)=0$.

It is therefore impossible to reach $100 \%$ specificity for RTE towards the lowest ADC values in the lung. Indeed, as demonstrated above, the lowest near-zero ADC values correspond to those of the normal lung parenchyma (see also figure 1). The ROC curve of the ADC indicates that the normal lung was excluded from this study. It seems unlikely that, by performing three random measurements inside the lung parenchyma of control subjects, such a systematic non-inclusion of any normal lung parenchyma may be reproducible. In our institution, we measured ADC values in $52 \mathrm{CF}$ patients, including those with RTE $(n=10)$ and those without RTE $(n=42)$ (mean age $=17 \pm 16$ years, male/female $=28 / 24)$. In agreement with the physics of MRI, we found, randomly, the lowest ADC values to be inside the predominant areas of normal lung parenchyma (at $0.2 \pm 0.3 \times 10^{-3} \mathrm{~mm}^{2} \cdot \mathrm{s}^{-1}$ ). Conversely, by selecting acute consolidations, we found the highest $\mathrm{ADC}$ values (see also figure 1).

@ERSpublications

Restriction of lung magnetic resonance diffusion is not specific for RTE but can correspond to a normal parenchyma http://ow.ly/wUgk30fDGQ8

Cite this article as: Dournes G, Laurent F. Restricted magnetic resonance diffusion of lung consolidation is not specific for respiratory exacerbation. Eur Respir J 2017; 50: 1701621 [https://doi.org/10.1183/ 13993003.01621-2017]. 

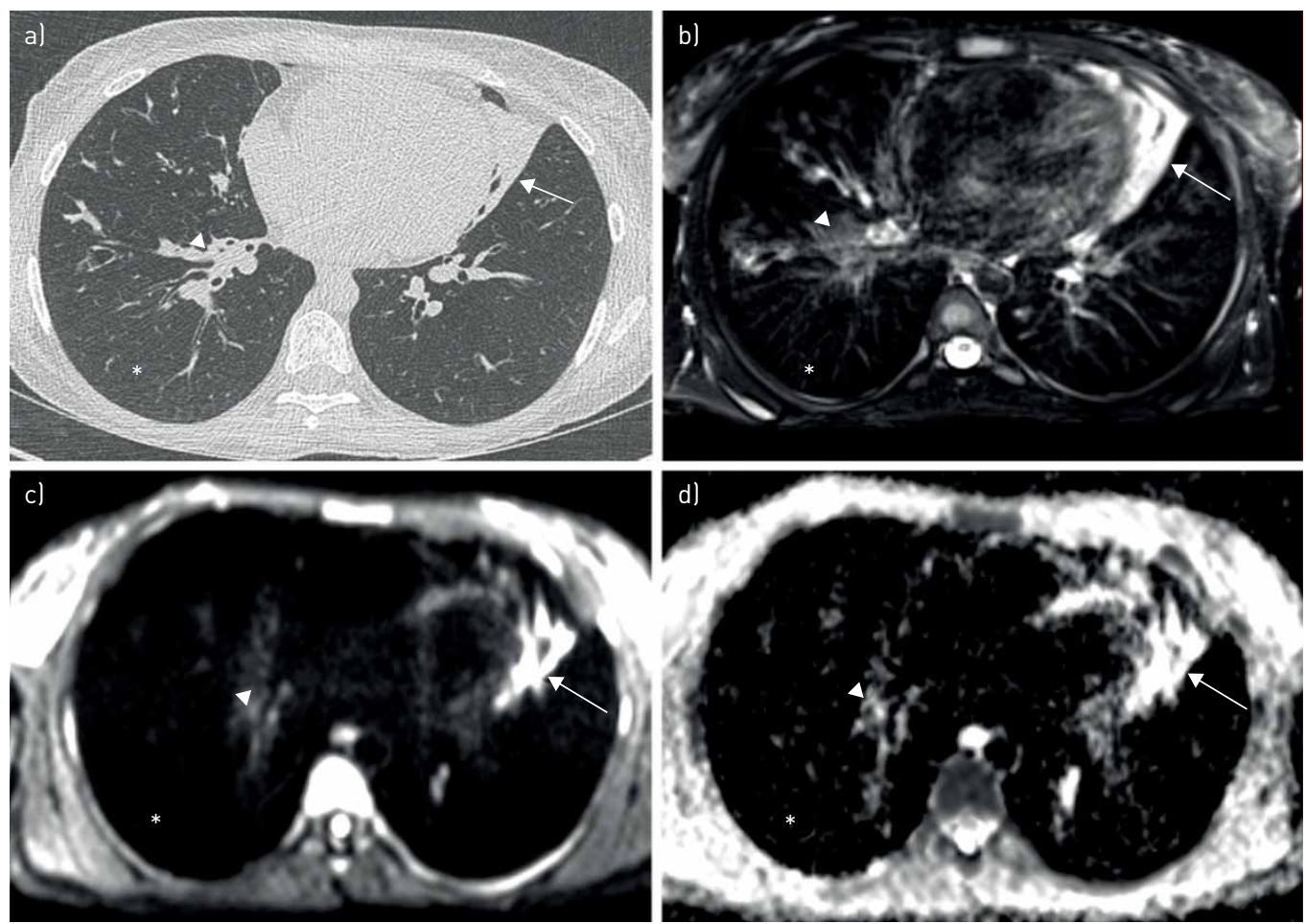

FIGURE 1 Computed tomography (CT) (a) and magnetic resonance imaging (MRI) images (b, $c, d$ ) using T2-weighting (b) and diffusion-weighting (DWI) (c), as well as the corresponding apparent diffusion coefficient (ADC) map reconstruction (d) in a 12-year-old cystic fibrosis (CF) patient undergoing respiratory tract exacerbation (RTE) before treatment. The white arrow indicates an area of newly developed consolidation, the white arrowhead an area of chronic bronchial wall thickening and the white star an area of normal lung parenchyma. The acute consolidation demonstrates both T2 signal hyper-intensity (b) and "hotspots" on the diffusion sequence (c). On the corresponding ADC map reconstruction (d) the area of consolidation appears bright whereas the normal lung parenchyma appears dark, demonstrating both high and low ADC values, respectively. In agreement with this visual record, the mean quantitative measurement of ADC in the area of consolidation is $2.2 \times 10^{-3} \mathrm{~mm}^{2} \cdot \mathrm{s}^{-1}$ compared to $0.2 \times 10^{-3} \mathrm{~mm}^{2} \cdot \mathrm{s}^{-1}$ in the normal lung parenchyma. ADC measurement in the area of chronic bronchial wall thickening is intermediate at $1 \times 10^{-3} \mathrm{~mm}^{2} \cdot \mathrm{s}^{-1}$ and stable as compared to previous yearly examinations. Both acute lung consolidation and chronic bronchial wall thickening display similar levels of increase under T2-weighting (b), diffusion-weighting (c) and ADC mapping (d) due to the T2-shine-through effect.

In the study by CIET et al. [1], the presence of abnormally elevated ADC only, in all CF controls with a DWI score of zero, suggests that the random exclusion of the normal lung induced a random diagnostic accuracy evaluation. However, this crucial information from normal lung parenchyma needs to be taken into account, because it yields important implications for therapeutic management. Indeed, in our institution, consolidations normally disappear under antibiotics in the vast majority of cases and thus the ADC values diminish towards those of normal lung parenchyma. In the CIET study, consolidation ADC values never reduce after treatment, indicating that the lesions remain abnormally uncleared (as confirmed by the morphology images). Finally, figures from the CIET article [1] demonstrate that the sternum, the cerebrospinal fluid and the ribs have prominent variations in DWI intensity before and after treatment. No signal variation is expected from these normal bone and water structures under antibiotics. Thus, the meaning of these variations, similar to those of the consolidations, is questionable.

To conclude, the study by CIET et al. [1] demonstrates higher DWI scores in CF patients to detect RTE but does not demonstrate that ADC is specifically reduced in RTE. Owing to the close similarity between DWI and T2 intensity variations (not only morphology), the T2-shine-through effect still remains a rationale to explain results from the DWI score $[2,3]$.

Gaël Dournes ${ }^{1,2}$ and François Laurent ${ }^{1,2}$

${ }^{1}$ Centre de Recherche Cardio-Thoracique de Bordeaux, INSERM U1045 (CIC 14.01), Université Bordeaux Segalen, Bordeaux, France. ${ }^{2}$ Service d'Imagerie Thoracique et Cardiovasculaire, Centre Hospitalier Universitaire (CHU) de Bordeaux, Pessac, France. 
Correspondence: Gaël Dournes, Centre de Recherche Cardio-Thoracique de Bordeaux, INSERM U1045, Université Bordeaux Segalen, 146 rue Léo Saignat, 33076 Bordeaux Cedex, France. E-mail: gael.dournes@chu-bordeaux.fr

Received: Aug 082017 | Accepted: Aug 142017

Conflict of interest: None declared.

\section{References}

1 Ciet $\mathrm{P}$, Bertolo S, Ros $\mathrm{M}$, et al. Detection and monitoring of lung inflammation in cystic fibrosis during respiratory tract exacerbation using diffusion-weighted magnetic resonance imaging. Eur Respir J 2017; 50: 1601437.

2 Cheng L, Blackledge MD, Collins DJ, et al. T2-adjusted computed diffusion-weighted imaging: A novel method to enhance tumour visualisation. Comput Biol Med 2016; 79: 92-98.

3 Vogel-Claussen J, Renne J, Hinrichs J, et al. Quantification of pulmonary inflammation after segmental allergen challenge using turbo-inversion recovery-magnitude magnetic resonance imaging. Am J Respir Crit Care Med 2014; 189: 650-657.

4 Biederer J, Beer M, Hirsch W, et al. MRI of the lung (2/3). Why ... when ... how? Insights Imaging 2012; 3: $355-371$.

5 Weller A, Papoutsaki MV, Waterton JC, et al. Diffusion-weighted (DW) MRI in lung cancers: ADC test-retest repeatability. Eur Radiol 2017; in press [https://doi.org/10.1007/s00330-017-4828-6].

6 Xin Y, Cereda M, Kadlecek S, et al. Hyperpolarized gas diffusion MRI of biphasic lung inflation in short- and long-term emphysema models. Am J Physiol Lung Cell Mol Physiol 2017; 313: L305-L312.

7 Gai ND, Malayeri AA, Bluemke DA. Long T2 suppression in native lung 3-D imaging using k-space reordered inversion recovery dual-echo ultrashort echo time MRI. MAGMA 2017; 30: 387-395.

8 Higano NS, Fleck RJ, Spielberg DR, et al. Quantification of neonatal lung parenchymal density via ultrashort echo time MRI with comparison to CT. J Magn Reson Imaging 2017; 46: 992-1000. 